\title{
A Simple Method for Static Analysis of Tubular High-Rise Buildings Using Timoshenko Beam Theory
}

\author{
Seyed Mozafar Davari ${ }^{1}$, Mohsen Malekinejad ${ }^{2}$, Reza Rahgozar ${ }^{3}$ \\ ${ }^{1}$ Ph.D. Candidate, Department of Civil Engineering, Sirjan Branch, Islamic Azad University, Sirjan, Iran. \\ sm_davari@iauk.ac.ir \\ ${ }^{2}$ Assistant Professor, Department of Civil Engineering, Sirjan Branch, Islamic Azad University, Sirjan, Iran. \\ m.malekinejad@iausirjan.ac.ir \\ ${ }^{3}$ Professor, Department of Civil Engineering, Shahid Bahonar University of Kerman, Kerman, Iran. \\ rahgozar@uk.ac.ir
}

\begin{abstract}
The paper proposed the continuum model, known as Kwan model, for the analysis of highrise buildings, which can be used as an appropriate approximation of the overall behaviour of the structure. In the proposed method, a high-rise building was modelled as a cantilever beam. Initially, the structure model was analysed with the assumption of flexural behaviour based on Euler-Bernoulli Beam Theory, and the displacement of floors was calculated. Then, in order to consider the shear lag effects in the overall displacement of the structure, Timoshenko's beam model was studied and related relations were extracted. The formulas obtained for the framed tube system, modelled with the Kwan beam, were calculated and the lateral displacement equation was measured. To verify the results, numerical models were created in software (ETABS) and statically were analysed for lateral loading, and finally the results were compared with the results of the analytical results and the corresponding diagrams were presented.
\end{abstract}

Keywords - Euler-Bernoulli's Beam Theory, Timoshenko's Beam Theory, Drift, Finite Element Method (FEM)

\section{INTRODUCTION}

The increase in the population and the reduction of useful space for life and business led the human to increase construction at the height, resulting in the idea of high-rise buildings. High-rise buildings are a logical and economic solution for the settlement of the population, jobs, departments, etc. in a small area of land, which can help the town planners, besides its appearance and glory. However, for many cosmopolitans, high-rise buildings are the only response to the continuous growth of population concentration. The purpose of building high-rise buildings is to use more area to meet the needs of different citizens. This is a way to save on land use and share prices to users, thus avoiding the unnecessary land use, coping with its scarcity, and expanding urban levels. The conventional analysis of tall structures is often time-consuming and costly due to having high degrees of freedom. The designers of this kind of buildings require a proper and reasonable design for their design and it is necessary to be able to analyze the static and dynamic characteristics of the structure quickly and accurately. Additionally, the effect of each variable in assumed structural system should be determined and finally, the first appropriate design should be recommended. Considering the importance of framed tube structures in high-rise buildings as a system resistant to lateral loads, it is necessary to have a simple but precise method for analyzing these structures.

Several methods have been proposed for framed-tubes analysis. Coull and Bose presented a method based on the theory of elasticity. In this method, the structure is equivalent to orthotopic planes, and the equations and equilibrium relations are satisfied in the equivalent structure [1].

Coull and Ahmed presented a method in calculation of stories displacement in the framed tube [2]. Kwan presented equations for determining the stress in columns and also obtaining the lateral displacement of the framed tube structure using equivalence orthotropic planes, energy relations and the theory of elasticity [3]. Connor and Pouangare recommended the five-stringer model, in which the structure is equalled with beams and vertical planes and by calculating the shear and moment rigidity of members, the formulas for stresses in the column are proceeded [4]. One of the other methods for improving the behavior of the framed tube is adding internal tubed frames to the original structure. In this case, the stress distribution and displacement will be appreciably reduced. Other methods for analyzing tubed frames are presented by researchers such as Paulino in 2010 [5], Mahjub et al. In 2011 [6], Kamgar and Rahgozar in 2013 [7], Rahgozar et al. in 2014 [8], Malekinejad and Rahgozar in 2014 [9], Khajuai Rad et al. in 2017 [10]. Davari and Rahgozar in 2019 conducted their research on the static analysis of high-rise buildings without shear lag effect, too [11].

In this paper, a simple approximation method have been proposed for static analysis of a tall structure with a framed tube system with symmetrical plan and fixed profile in height using a Timoshenko beam model with regard to shear and flexural hardening and regardless of the shear lag effects. 
In this method, the framed tube was equated with four orthotropic orthogonal planes with a hollow box section and the structure of the framed tube was placed under a uniform, triangular and concentrated load was placed at the end of the structure and static analysis was performed.

\section{EULER-BERNOULLI'S THEORY}

Research development has been seen in this area in the light of the importance of using thin-walled beams in structure designing during recent decades.

Bending thin-walled beam is modelled by Euler-Bernoulli theory in most of these studies. The first theory in this field is elementary beam theory based on Euler-Bernoulli theory, which is the flatness of the planes perpendicular to the longitudinal axis of the beam before and after bending. This theory has proper accuracy for thin beams and the beams under pure bending.

The hypotheses of the Euler-Bernoulli theory are as follows:

1) Perpendicularity of the planes to the neutral axis;

2) Each plane remains as a plane.

3) The beam is placed under pure bending.

4) The beam section is constant and symmetric.

5) The stress-strain relationship follows Hooke's law.

The assumption of beam section's remaining plane after bending is one of the most applied and important assumptions introduced in solid mechanics so far. The deformation of a beam is shown in Fig. 1. Elementary beam theory is based on this assumption and is an accurate solution for pure bending. However, if the beam is placed under another loading, this hypothesis will not have high reliability.

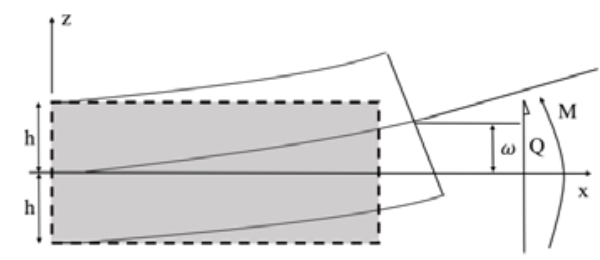

Fig. 1. Deformation of Euler- Bernoulli's beam

As it is seen from the geometry of Fig. 2, the following equation will be obtained:

$$
\begin{gathered}
u_{x}=-z \varphi(x) \\
\varphi(x)=\frac{d \omega}{d x}
\end{gathered}
$$

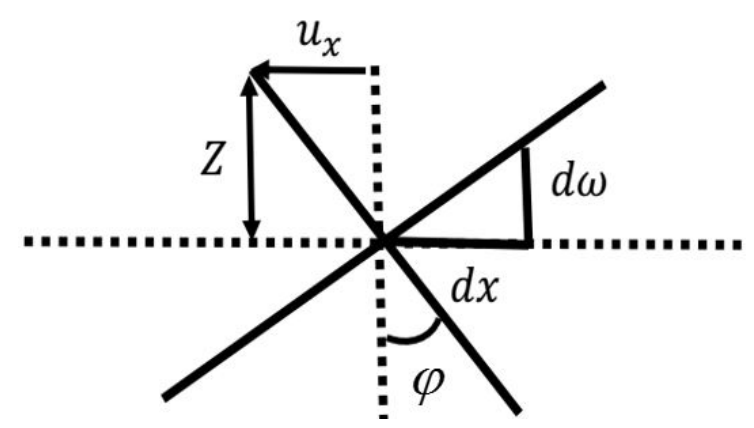

Fig. 2. Deformation geometry in the Euler-Bernoulli's theory

Where the longitudinal displacement of each point is $u_{x}, \omega$ is the displacement of this plane in $z$ direction, $\varphi$ is the angle of the plane, which shows the rotation of the plane after bending and $z$ is the distance from the neutral axis.

The shear strain is obtained according to the following formula:

$$
\gamma_{x z}=\frac{d u_{x}}{d z}+\frac{d u_{z}}{d x}=-\varphi+\frac{d \omega}{d x}=0
$$


According to Fig. 2, the other side of equation will be written and it is understood that these two equations are the same but symmetrical, so:

$$
\tau_{x z}=G \gamma_{x z}=G \times 0=0
$$

As can be seen, the shear stress and strain are zero, which proves Euler-Bernoulli assumption.

And by using Hooke's law:

$$
\sigma_{x}=E \varepsilon_{x}
$$

On the other hand, it can be written:

$$
\varepsilon_{x}=\frac{d u_{x}}{d x}=-z d \varphi(x)=-z \frac{d^{2} \omega}{d x^{2}}
$$

Using the equilibrium equation, the structure moment can be obtained as follows:

$$
M_{x}=-\int \sigma_{x} \times z d A=\int E z^{2} \frac{d^{2} \omega}{d x^{2}} \times d A=E \frac{d^{2} \omega}{d x^{2}} \int z^{2} d A=E I \frac{d^{2} \omega}{d x^{2}}
$$

\section{A. Uniform Loading Distribution}

Fig. 3 indicates a high-rise building with a framed-tube system which is modeled as a cantilever beam, and is under uniform loading distribution $q$. The rotation of the structure is assumed $\varphi$ and lateral displacement is shown by $\omega$.

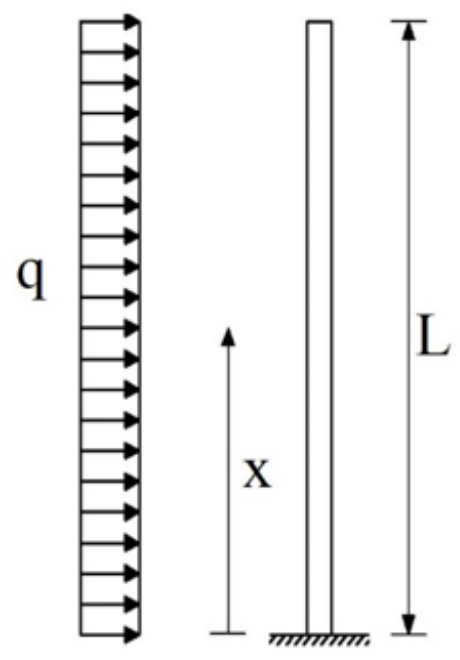

Fig. 3. Structure analysis model under uniform loading pattern

Based on the strength of material and analysis of structures, moment equation in the section at the distance $\mathrm{x}$ from the support is like Eq. 7:

$$
M_{x}=\frac{q L^{2}}{2}-q L x+\frac{q x^{2}}{2}
$$

Also, by combining Eq. 6 and Eq. 7 :

$$
E I \frac{d^{2} \omega}{d x^{2}}=\frac{q L^{2}}{2}-q L x+\frac{q x^{2}}{2}
$$

In Eq. 8, $\mathrm{L}$ is the length of the beam. Based on Eq. 1 and applying integral on it, Eq. 9 could be developed:

$$
\varphi(x)=\frac{d \omega}{d x}=\frac{q}{E I}\left(\frac{L^{2} x}{2}-\frac{L x^{2}}{2}+\frac{x^{3}}{6}+C_{1}\right)
$$

By applying integral on Eq. 9, Eq. 10 is proceeded:

$$
\omega(x)=\frac{q}{E I}\left(\frac{L^{2} x^{2}}{4}-\frac{L x^{3}}{6}+\frac{x^{4}}{24}+C_{1} x+C_{2}\right)
$$


And by boundary conditions that is shown in Eq. 11, the constants would be:

$$
\begin{aligned}
& \omega(0)=0 \quad \rightarrow \quad C_{2}=0 \\
& \varphi(0)=0 \quad \rightarrow \quad C_{1}=0
\end{aligned}
$$

Using the above result, formulas of Eq. 9 and Eq. 10 are simplified as follows:

$$
\begin{gathered}
\omega(x)=\frac{q}{E I}\left(\frac{L^{2} x^{2}}{4}-\frac{L x^{3}}{6}+\frac{x^{4}}{24}\right) \\
\varphi(x)=\frac{d \omega}{d x}=\frac{q}{E I}\left(\frac{L^{2} x}{2}-\frac{L x^{2}}{2}+\frac{x^{3}}{6}\right)
\end{gathered}
$$

\section{B. Triangular Loading Distribution}

When the structure is subjected to a triangular loading pattern in accordance with Fig. 4, the angle of rotation of the different points at the height of the structure is equal to $\varphi$ and the lateral displacement is equal to $\omega$.

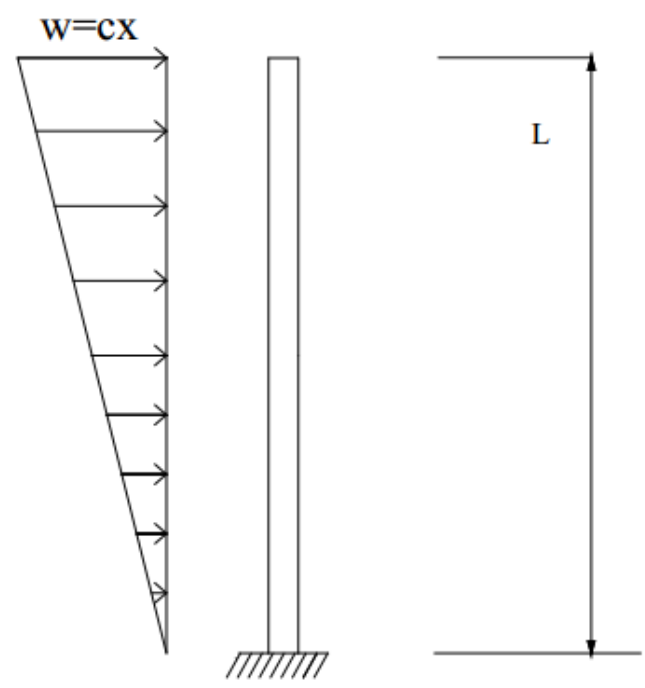

Fig. 4. Analysis model of structure under triangular loading pattern

In this loading pattern, $M_{x}$ will be changed into Eq. 14:

$$
M_{x}=\frac{c L^{2} x}{2}-\frac{c L^{3}}{3}-\frac{c x^{3}}{6}
$$

By using Eq. 6 and integral of Eq. 14, Eq. 15, it will be concluded:

$$
\begin{aligned}
& E I \frac{d^{2} \omega}{d x^{2}}=\frac{c L^{2} x}{2}-\frac{c L^{3}}{3}-\frac{c x^{3}}{6} \\
& \varphi(x)=\frac{d \omega}{d x}=\frac{c}{E I}\left(\frac{L^{2} x^{2}}{4}-\frac{L^{3} x}{3}-\frac{x^{4}}{24}\right)+c_{1}
\end{aligned}
$$

By applying boundary condition on Eq. 15, the constant will be:

$$
\varphi(0)=0 \Rightarrow c_{1}=0
$$

So:

$$
\varphi(x)=\frac{c}{E I}\left(\frac{L^{2} x^{2}}{4}-\frac{L^{3} x}{3}-\frac{x^{4}}{24}\right)
$$

By using Eq. 17, $\omega(x)$ will be calculated in Eq. 18:

$$
\begin{aligned}
& \omega(x)=\int \varphi(x) d x \\
& \omega(x)=\frac{c}{E I}\left(\frac{L^{2} x^{3}}{12}-\frac{L^{3} x^{2}}{6}-\frac{x^{5}}{120}\right)+c_{1}
\end{aligned}
$$


By applying another boundry condition, the constant of integration will be obtained:

$$
\omega(0)=0 \quad \Rightarrow \quad c_{1}=0
$$

Overall, the displacement formula will proceed in Eq. 20:

$$
\omega(x)=\frac{C}{E I}\left(\frac{L^{2} x^{3}}{12}-\frac{L^{3} x^{2}}{6}-\frac{x^{5}}{120}\right)
$$

\section{Concentrated Force at the Tip of the Structure}

In this section, the rotation and the lateral displacement will be $\varphi$ and $\omega$, respectively.
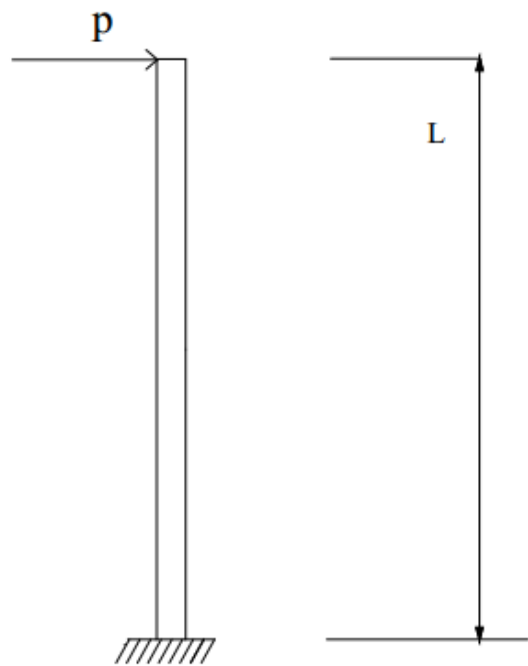

Fig. 5. Analysis model of structure under concentrated force at the tip of the structure

In this situation, $\mathrm{M}_{\mathrm{x}}$ formula will be like Eq. 21 and the other relationships will be concluded like previous, so:

$$
\begin{aligned}
& M_{x}=P L-P x \\
& M_{x}=E I \frac{d^{2} \omega}{d x^{2}} \\
& E I \frac{d^{2} \omega}{d x^{2}}=P L-P x \\
& \varphi(x)=\frac{d \omega}{d x}=\frac{P}{E I}\left(L x-\frac{x^{2}}{2}\right)+c_{1}
\end{aligned}
$$

By applying boundary condition, the constant of the integration will be obtained in Eq. 22:

$$
\varphi(0)=0 \quad \Rightarrow \quad c_{1}=0
$$

Moreover, using integral on Eq. 21, the $\omega(x)$ will be:

$$
\omega(x)=\frac{P}{E I}\left(\frac{L x^{2}}{2}-\frac{x^{3}}{6}\right)+c_{1}^{\prime}
$$

By using another boundary condition, the constant of integration will be obtained as in Eq. 24:

$$
\omega(0)=0 \quad \Rightarrow \quad c_{1}^{\prime}=0
$$

Then, the formulae of this part has been written as Eq. 25:

$$
\begin{aligned}
& \varphi(x)=\frac{d \omega}{d x}=\frac{P}{E I}\left(L x-\frac{x^{2}}{2}\right) \\
& \omega(x)=\frac{P}{E I}\left(\frac{L x^{2}}{2}-\frac{x^{3}}{6}\right)
\end{aligned}
$$




\section{RECOMMENDED METHOD}

The recommended method is Timoshenko's beam theory. In Euler-Bernoulli beam method, the shear effect is neglected, but in deep beams, the effect of shear strain is tangible, so Euler-Bernoulli method is not a comprehensive criterion. The proposed method was based on Timoshenko's beam theory to solve this problem. In the following case, the review assumptions used in this analysis are as follows:

1. Each plane remains as plain.

2. The beam section area is constant and symmetric.

3. The stress-strain equation follows Hook's law.

As can be seen in above hypotheses, the condition of the perpendicularity of the plane is removed from Timoshenko's beam theory (Fig. 6).

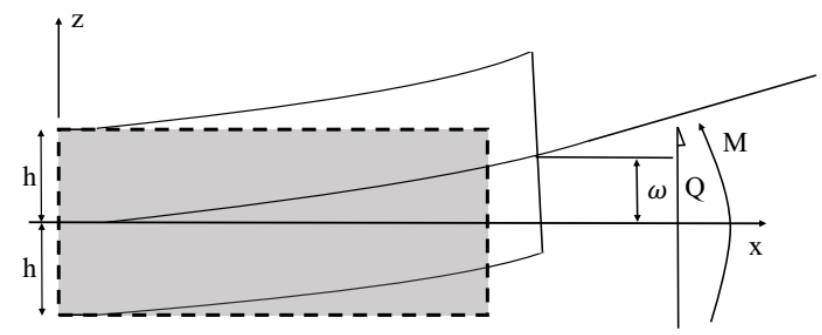

Fig. 6. Deformation of the Timoshenko's beam

According to Fig. 6, Eq. 26 will be developed:

$$
\begin{aligned}
& u_{x}=-z \varphi(x) \\
& \varepsilon_{x}=\frac{d u_{x}}{d x}=-z \frac{d \varphi(x)}{d x}
\end{aligned}
$$

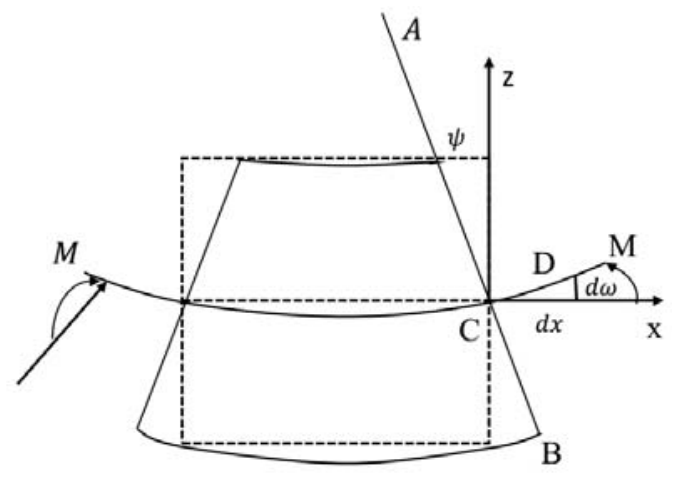

Fig. 7. The geometry of the deformation of Timoshenko's beam theory

By Hook's law and Eq. 26, Eq. 27 will be proceeded:

$$
\sigma_{x}=-E z \frac{d \varphi(x)}{d x}
$$

On the other hand, the shear strain will be calculated by Eq. 28:

$$
\gamma_{x z}=\frac{d \omega}{d x}-\varphi(x)
$$

To calculate the shear stress based on formula $\tau_{x z}=\gamma_{x z} G$, the shear stress will be obtained by Eq. 29:

$$
\tau_{x z}=\left(\frac{d \omega}{d x}-\varphi(x)\right) G
$$

This theory is more precise than Euler-Bernoulli's theory, but due to constant shear strain distribution at the height, the shear correction factor $k$ will be used in Eq. 30, so:

$$
\tau_{x z}=k G\left(\frac{d \omega}{d x}-\varphi(x)\right)
$$


Eq. 31 shows the bending moment equilibrium equation:

$$
M_{x}=-\int z \sigma_{x} d A
$$

Using Eq. 27 and Eq. 31, the moment of the shear will be:

$$
M_{x}=\int E z^{2} \frac{d \varphi(x)}{d x} d A=E I \frac{d \varphi(x)}{d x}
$$

The shear force based on $\tau_{x z}$ is like Eq. 33:

$$
V_{x}=\int \tau_{x z} d A
$$

And by applying Eq. 30 on Eq. 33, the shear force will be:

$$
V_{x}=\int k G\left(\frac{d \omega}{d x}-\varphi(x)\right) d A=k G A\left(\frac{d \omega}{d x}-\varphi\right)
$$

In the above formulae, $G$ and $k$ are the beam shear module and the shear correction factor, respectively, which is explained well in references [3] and [11]. Additionally, $A$ is the effective area in shear at the section of the framed-tube.

On the other hand, the equalled moment of inertia of the framed-tube section, presented in the Fig. 8, will be proceeded in Eq. 35:

$$
I=\frac{1}{12}(2 b)(2 a+2 t)^{3}-\frac{1}{12}(2 b-2 t)(2 a)^{3}=4 a^{2} b t+\frac{4}{3} a^{3} t
$$

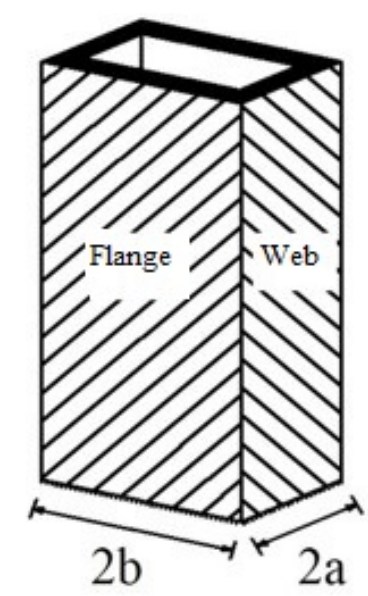

Fig. 8. The Equilibrium structure

By excluding the powers above one of $t$ in Eq. 35, Eq. 36 will be generated, in which $t$ is the equivalent thickness of the plan:

$$
t=\frac{A_{c}}{S}
$$

Where $A_{c}$ and $S$ are the column section area and the distance of the column axis, respectively.

\section{A. Uniform Loading Pattern}

When the structure is placed under uniform loading pattern with $q$ intensity like Fig. 9, the rotation angle of the beam at the end of it will be $\varphi$ and the displacement of it will equal to $\omega$. 


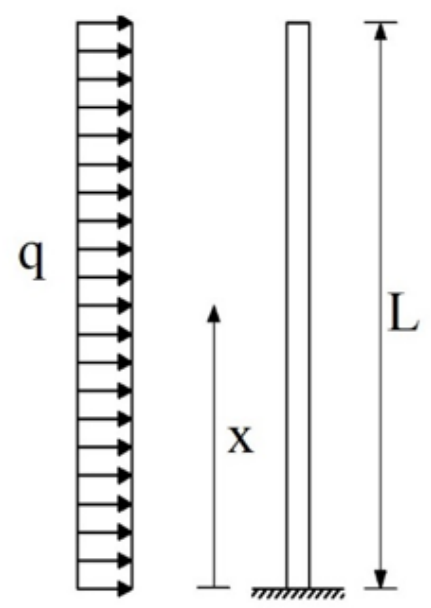

Fig. 9. Timoshenko's beam model under uniform loading pattern

In this form of loading, moment equation in the distance of $\mathrm{x}$ from support will proceed like Eq. 37 :

$$
M_{x}=\frac{q L^{2}}{2}-q L x+\frac{q x^{2}}{2}
$$

By using equation $M_{x}=E I \frac{d \varphi}{d x}$ in Eq. 37 :

$$
E I \frac{d \varphi}{d x}=\frac{q L^{2}}{2}-q L x+\frac{q x^{2}}{2}
$$

By using integral, Eq. 39 will be resulted from Eq. 38:

$$
\varphi(x)=\frac{q}{E I}\left(\frac{L^{2} x}{2}-\frac{L x^{2}}{2}+\frac{x^{3}}{6}\right)+C_{1}
$$

By applying boundary condition, the constant will be calculated:

$$
\varphi(0)=0 \quad \Rightarrow \quad \mathrm{C}_{1}=0
$$

Based on Eq. 35, shear will proceed in Eq. 41:

$$
V(x)=k A G\left(\frac{d \omega}{d x}-\varphi(x)\right)
$$

By using Eq. 41, Eq. 42 will be developed:

$$
\frac{d \omega}{d x}=\frac{V(x)}{k A G}+\varphi(x)
$$

By applying integral on Eq. 42 and using boundary conditions, the constant will be obtained:

$$
\begin{aligned}
& \omega(x)=\int \frac{d \omega}{d x}=\int \varphi(x) d x+\int \frac{V(x)}{k A G} d x+C_{1} \\
& \omega(0)=0 \rightarrow C_{1}=0
\end{aligned}
$$

The Eq. 43 will be simplified to Eq. 44 :

$$
\begin{aligned}
\omega(x) & =\int \frac{d \omega}{d x}=\int \varphi(x) d x+\int \frac{V(x)}{k A G} d x \\
& =\frac{q}{E I}\left(\frac{L^{2} x^{2}}{4}-\frac{L x^{3}}{6}+\frac{x^{4}}{24}\right)+\frac{q L x-\frac{q x^{2}}{2}}{k A G}
\end{aligned}
$$

\section{B. Triangular Loading Pattern}

In this section, the model of structure under triangular loading pattern equals with $w=c X$, which could be understood from Fig. 10. 

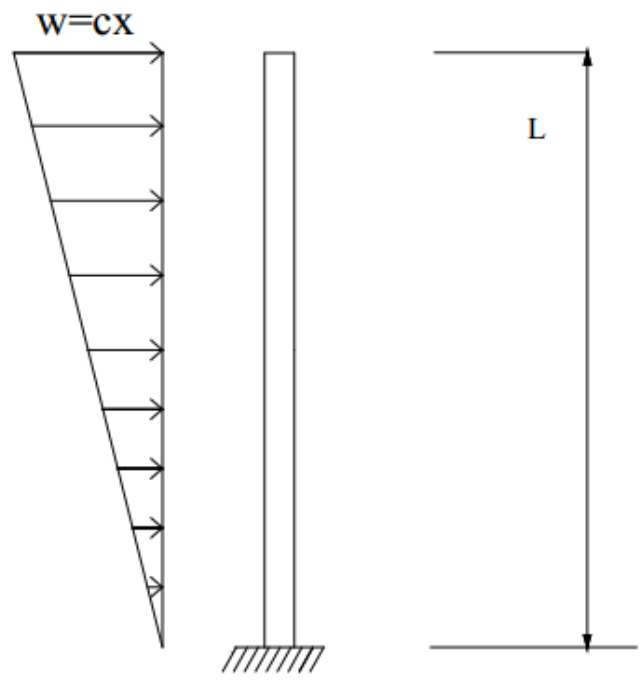

Fig. 10. Timoshenko's beam modelling under triangular loading pattern

In this type of loading pattern, the moment equation will be like Eq. 45 :

$$
M_{x}=\frac{c L^{2} X}{2}-\frac{c L^{3}}{3}-\frac{c x^{3}}{6}
$$

As a result, based on formula $M_{x}=E I \frac{d \varphi}{d x}$ in Eq. 45, Eq. 46 will be resulted:

$$
E I \frac{d \varphi}{d x}=\frac{c L^{2} x}{2}-\frac{c L^{3}}{3}-\frac{c x^{3}}{6}
$$

By integration of Eq. 46, Eq. 47 was developed:

$$
\varphi(x)=\frac{c}{E I}\left(\frac{L^{2} x^{2}}{4}-\frac{L^{3} x}{3}-\frac{x^{4}}{24}\right)+c_{1}
$$

By applying the boundary condition, which is:

$$
\varphi(0)=0 \quad \Rightarrow \quad c_{1}=0
$$

As a result:

$$
\varphi(x)=\frac{c}{E I}\left(\frac{L^{2} x^{2}}{4}-\frac{L^{3} x}{3}-\frac{x^{4}}{24}\right)
$$

By presented explanation, shear and lateral displacement will be such as Eq. 50 and Eq. 51:

$$
\begin{gathered}
V(x)=\frac{C}{2}\left(L^{2}-x^{2}\right) \\
\omega(x)=\frac{c}{E I}\left(\frac{L^{2} x^{3}}{12}-\frac{L^{3} x^{2}}{6}-\frac{x^{5}}{120}\right)+c_{1} \\
+\frac{c}{2 k A G}\left(L^{2} x-\frac{x^{3}}{3}\right)+c_{2}
\end{gathered}
$$

By applying boundary conditions in Eq. 51, which is:

$$
\omega(0)=0 \quad \Rightarrow \quad c_{1}=c_{2}=0
$$


As a result:

$$
\begin{aligned}
\omega(x) & =\frac{c}{E I}\left(\frac{L^{2} x^{3}}{12}-\frac{L^{3} x^{2}}{6}-\frac{x^{5}}{120}\right)+ \\
& +\frac{c}{k A G}\left(\frac{x^{4}}{24}+\frac{L^{3} x}{3}-\frac{L^{2} x^{2}}{4}\right)
\end{aligned}
$$

C. Concentrated Force at the Tip of the Structure

In this occasion, the structure will be placed under concentrated force at the tip of the structure like Fig. 11.

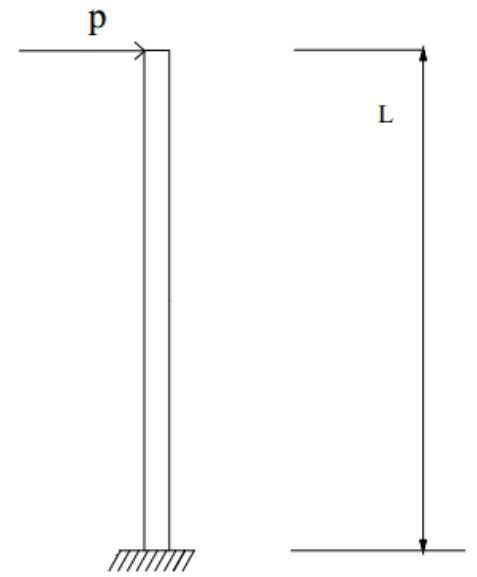

Fig. 11. Timoshenko's beam modelling under concentrated force at the tip of the structure.

In this situation, $\mathrm{M}_{\mathrm{x}}$ formula will be like Eq. 54:

$$
M_{x}=P L-P x
$$

By using $M_{x}=E I \frac{d \varphi(x)}{d x}$, Eq. 55 proceeds based on Eq. 54:

$$
E I \frac{d \varphi(x)}{d x}=\frac{P}{E I}(L-x)
$$

By integrating the above relation and applying boundary conditions, the following equation is resulted:

$$
\varphi(x)=\frac{P}{E I}\left(L x-\frac{x^{2}}{2}\right)
$$

By following the process presented in the previous sections, it is concluded that:

$$
\begin{aligned}
& V(x)=k A G\left(\frac{d \omega}{d x}-\varphi(x)\right) \\
& V(x)=P \\
& \omega(x)=\int \varphi(x) d x+\int \frac{V(x)}{k A G} d x \\
& \omega(x)=\frac{P}{E I}\left(\frac{L x^{2}}{2}-\frac{x^{3}}{6}\right)+\frac{P}{k A G} x+c_{1}^{\prime}
\end{aligned}
$$

And by applying other boundary conditions:

$$
\omega(0)=0 \quad \Rightarrow \quad c_{1}^{\prime}=0
$$

As a result:

$$
\omega(x)=\frac{P}{E I}\left(\frac{L x^{2}}{2}-\frac{x^{3}}{6}\right)+\frac{P}{k A G}\left(L x-\frac{x^{2}}{2}\right)
$$




\section{COMPARISON}

In the following, two examples are presented to examine the proposed method and compare the results of numerical investigation with two others theories. Each of these structures has been analysed and studied with finite element method, proposed method and Euler-Bernoulli's theory under a uniform static load pattern.

A. Concrete Framed-Tube with Symmetric Plan

The structure of a 40 -story concrete frame with a symmetrical plan has been used and statically analysed (Fig. 12) [3]. The overall dimensions of the structure are $35 \times 30 \mathrm{~m}^{2}$. The distance between the columns is $2.5 \mathrm{~m}$ and the height of each floor is $3 \mathrm{~m}$. In this structure, the dimensions of all beams and columns are $0.8 \times 0.8 \mathrm{~m}^{2}$ and the Young modulus and the shear modulus of the materials are $200 \mathrm{GPa}$ and $8 \mathrm{GPa}$, respectively. In addition, a uniform loading pattern $(120 \mathrm{kN} / \mathrm{m})$ is applied to the structure to calculate the deformation of the structure.

For numerical analysis, we consider the values of some parameters given for a Timoshenko beam in a uniformly loading pattern, as the following. The parameter $\mathrm{t}$, and $\mathrm{A}$ and $\mathrm{S}$ were defined in Eq. 36 as well. I is the section modulus of the equivalent section. $\mathrm{k}$ is the shape factor of the box section. $\Delta_{\mathrm{b}}$ and $\Delta_{\mathrm{c}}$ are the deflection induced by bending and the shear, respectively. $\mathrm{Q}$ is defined as lateral loading pattern, too. Moreover, the all parameters are described in reference [11]. The mentioned parameters will be equal to Table I.

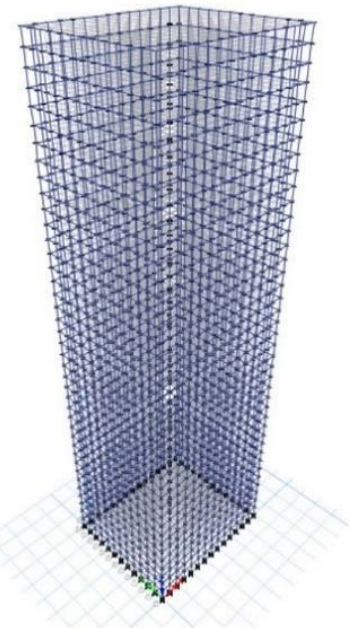

(a)

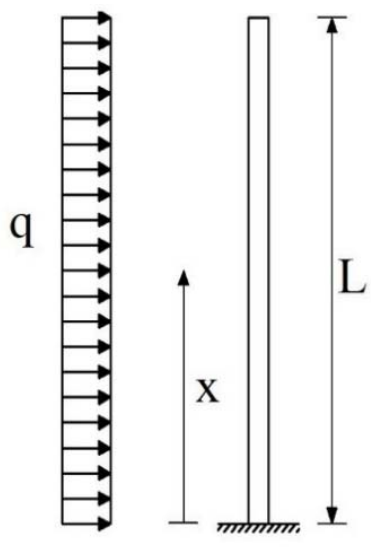

(b)

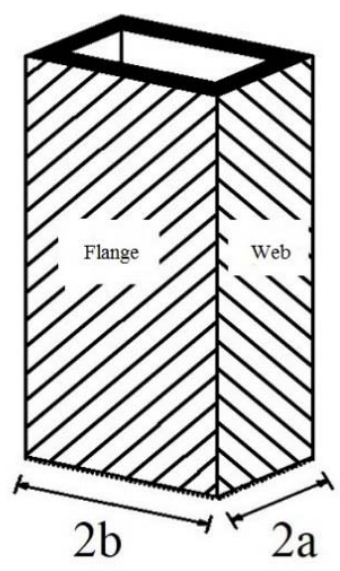

(c)

Fig. 12. The framed-tube system: a) The real structure; b) the behaviour of the high-rise building against the lateral loads; and c) The equivalent structure

Table I. The Parameters of Equivalent Structure

\begin{tabular}{cccccc}
$t=\frac{A_{c}}{S} \quad I=4 a^{2} b t+\frac{4}{3} a^{3} t$ & $k$ & $\frac{\Delta_{b}}{Q}=\frac{\left(h-d_{b}\right)^{3}}{12 E_{m} I_{c}}+\left(\frac{h}{S}\right)^{2} \frac{\left(S-d_{c}\right)^{2}}{12 E_{m} I_{b}}$ & $\frac{\Delta_{s}}{Q}=\frac{\left(h-d_{b}\right)}{G_{m} A_{s c}}+\left(\frac{h}{S}\right)^{2} \frac{\left(S-d_{c}\right)}{G_{m} A_{s b}}$ & $G=\frac{\frac{h}{S t}}{\frac{\Delta_{b}}{Q}+\frac{\Delta_{s}}{Q}}$ \\
\hline $0.26 \mathrm{~m}$ & $5184 \mathrm{~m}^{4}$ & $\frac{5}{6}$ & $2.1634 \times 10^{-9}$ & $0.9078 \times 10^{-9}$ & $1.526 \mathrm{GPa}$ \\
\hline
\end{tabular}

In accordance with Eq. 44, the displacement of stories in this case is obtained from the following equation:

$$
\omega(x)=\frac{q}{E I}\left(\frac{L^{2} x^{2}}{4}-\frac{L x^{3}}{6}+\frac{x^{4}}{24}\right)+\frac{q l x-q x^{2} / 2}{k A G}
$$

\section{RESULTS}

To compare the results of above methods, including ETABS, Euler-Bernoulli and Timoshenko methods, once a 40-storey concrete structure with symmetric plan and then an 80-storey like previous one had been modelled in ETABS and the displacement of the tip of the structure had been compared. Fig. 13 shows the displacement of stories for the three proposed methods of a 40-storey building.

Based on Fig. 13, the differential displacement of the last story by the FEM and the Bernoulli method is about $77 \%$, though it is only $13 \%$ for the FEM and the Timoshenko beam theory.

Fig. 14 shows the stories displacement for the three proposed methods for an 80 -story building with the same properties of the previous structure. The final displacement of Bernoulli's method differs from the displacement of ETABS to $46 \%$, which is about $10 \%$ for the proposed method with finite element method. 


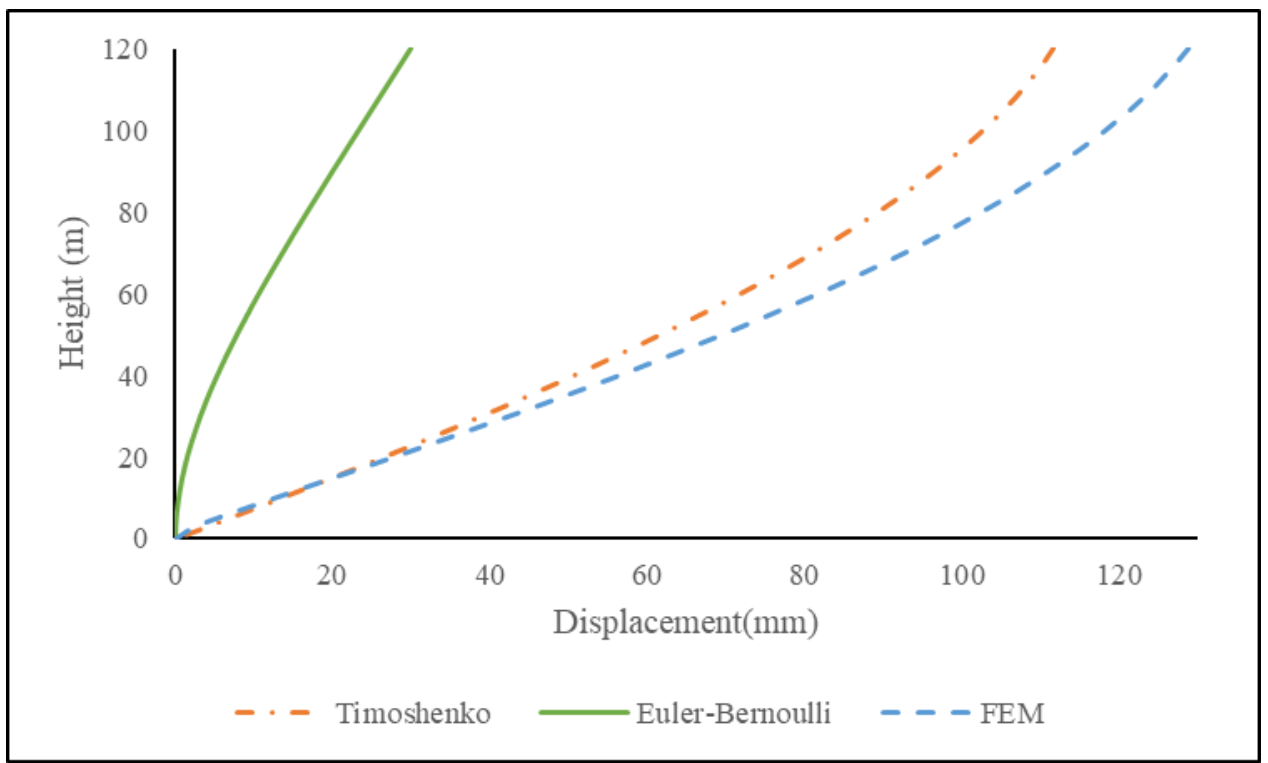

Fig. 13. Comparison of the displacement obtained from the three proposed methods for a 40-story concrete building

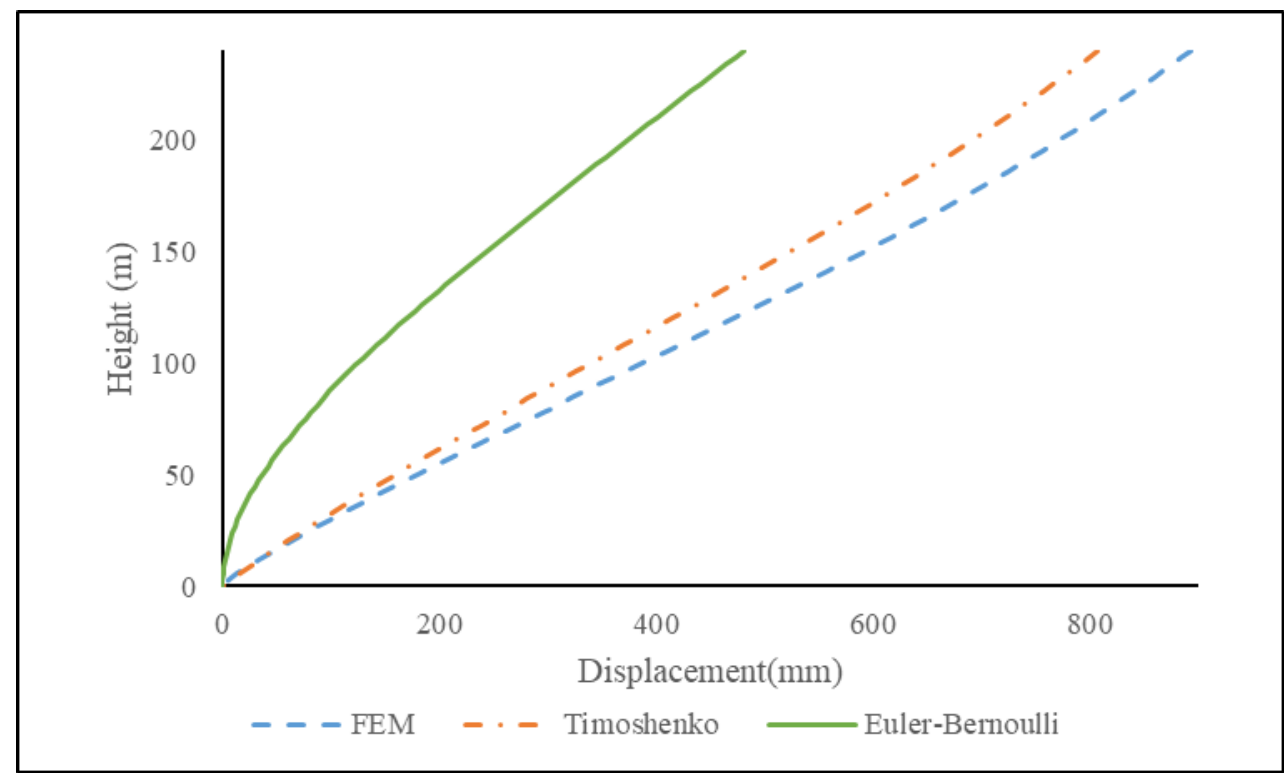

Fig. 14. Comparison of the displacement obtained from the three proposed methods for an 80-story concrete building

As it is clear from the Figs. 13 and 14, the Timoshenko beam theory has an acceptable approximation until 30 $\mathrm{m}$ height, which it could be concluded that it is useful for the nearly 10 stories buildings.

\section{CONCLUSION}

- The proposed model for the analysis of tall structures with symmetrical plan produced acceptable results. In particular, in the initial design phase, this method could provide a proper prediction of the behavior of the structure.

- Although numerical methods and commercial software were more accurately able to model tall structures, the analytical approach presented in the proposed method provided the ability to determine the parameters affecting structure behavior and their sensitivity analysis in the structure response.

- The proposed method was less costly compared to the numerical methods such as finite element, and required less computing procedures. This issue becomes more important in the case of high-rise structures made up of a large number of elements.

- The results of the analysis presented which shear effect is considerable and because of that, the Timoshenko theory adapted vigorously on the FEM results.

- The Timoshenko beam theory can predict the displacement of the structures with the lower $30 \mathrm{~m}$ height and its conclusion has suitable accuracy.

- By the increase in the height of the structure, The Timoshenko beam theory had lower differential with the FEM. 


\section{REFERENCES}

[1] Coull A., Bose B. Simplified analysis of frame tube structures. Journal of Structural Division, 101: 2223-2240, 1975.

[2] Coull A., Ahmed K. Deflection of framed-tube structures, Journal of Structural Engineering, ASCE, 104: 857-862, 1978.

[3] Kwan AKH. Simple method for approximate analysis of framed-tube structures. Journal of Structural Engineering, ASCE, 120: 12211239, 1994.

[4] Connor JJ., Pouangare CC. Simple model for design of framed tube structures. Journal of Structural Engineering, ASCE, 117: 3623$3644,1991$.

[5] Paulino MR. Preliminary Design of Tall Buildings. M.Sc. Thesis, Civil and Environmental Engineering Department, Worcester Polytechnic Institute, Worcester, Massachusetts, USA, 2010.

[6] Mahjoub R., Rahgozar R., Saffari H. Simple method for analysis of tube frame by consideration of negative shear lag. Australian Journal of Basic and Applied Sciences. 5(3): 309-316 2011

[7] Kamgar R., Rahgozar R. A simple approximate method for free vibration analysis of framed tube structures. The Structural Design of Tall and Special Buildings. John Wiley and Sons. 22: 217-234, 2013.

[8] Rahgozar R., Ahmadi A. R., Ghelichi M., Goudarzi Y., Malekinejad M., Rahgozar P. Parametric stress distribution and displacement functions for tall buildings under lateral loads. The Structural Design of Tall and Special Buildings Journal, John Wiley and Sons, 23: 22-41, 2014

[9] Malekinejad M., Rahgozar R. An analytical model for dynamic response analysis of tubular tall buildings. The Structural Design of Tall and Special Buildings Journal, John Wiley and Sons, 23: 67-80, 2014.

[10] Khajouyi Rad M., Alavi S., Rahgozar R. Optimum location of outrigger-belt truss in tall buildings based on energy method in hybrid systems. The $5^{\text {th }}$ International Congress of Civil Engineering, Shahid Beheshti University, Tehran, 2017.

[11] Davari. S.M., Rahgozar R. The static analysis of high-rise buildings with framed tube system based on Timoshenko beam theory without shear lag effect. The $4^{\text {th }}$ International Conference on Urban Management, Civil Engineering and Technology in Modern Architecture, Paris, 2019. 